\title{
No evidence of a strong magnetic field in the Blazhko star RR Lyrae ${ }^{\star}$
}

\author{
M. Chadid ${ }^{1}$, G. A. Wade ${ }^{2}$, S. L. S. Shorlin ${ }^{3}$, and J. D. Landstreet ${ }^{3}$ \\ 1 Observatoire de la côte d'Azur, UMR 6528, BP 4229, 06304 Nice Cedex 04, France \\ 2 Department of Physics, Royal Military College of Canada, PO Box 17000, Station "Forces", Kingston, Ontario, \\ Canada K7K 4B4 \\ ${ }^{3}$ Department of Physics \& Astronomy, the University of Western Ontario, London, Ontario, Canada N6A 3K7
}

Received 9 April 2003 / Accepted 9 October 2003

\begin{abstract}
In this paper we report a new series of high-precision (median $\sigma_{\mathrm{B}} \sim 80 \mathrm{G}$ ) longitudinal magnetic field measurements of RR Lyrae, the brightest Blazhko star, obtained with the MuSiCoS spectropolarimeter over a period of almost 4 years from 1999-2002. These data provide no evidence whatsoever for a strong magnetic field in the photosphere of RR Lyrae, a result consistent with Preston's (1967) results, but inconsistent with apparent magnetic field detections by Babcock (1958) and Romanov et al. (1987, 1994). Following discussion of these disparate results, we conclude that RR Lyrae is a bona fide non-magnetic star, a conclusion which leads to the general falsification of models of the Blazkho effect requiring strong photospheric magnetic fields. Furthermore, due to the 4 year baseline of our observations, we can also dismiss the hypothesis that RR Lyrae undergoes a 4-year "magnetic cycle". Therefore the origin of the observed 4-year modulation of RR Lyrae remains unexplained, and we stress the need for additional theoretical efforts to come to a better understanding of this phenomenon.
\end{abstract}

Key words. stars: variables: RR Lyr - stars: oscillations - stars: magnetic fields - stars: individual: RR Lyrae techniques: spectroscopic, polarimetric

\section{Introduction}

RR Lyrae stars have contributed to almost every branch of modern astronomy. They are standard candles, witnesses of the evolution of the universe at a young age, and prototypes of pure radial pulsators. Their photometric and spectroscopic properties have been studied for a century. However, up to now magnetic field measurements have yielded ambiguous results. The investigation of the magnetic fields of RR Lyrae-type pulsating stars is extremely important for understanding the nature of pulsations, and more particularly for understanding the physical origin of the Blazhko effect (Blazhko 1907), a periodic amplitude modulation shown by some $20-30 \%$ of the RRab stars, and about $2 \%$ of the RRc stars (Kovàcs 2001). This effect is a small but definite periodic variation in both the amplitude and the phase of pulsation observed in many RR Lyrae stars, which in RR Lyrae itself has a period of $40.8 \mathrm{~d}$ (for comparison, the pulsation period is $0.567 \mathrm{~d}$ ). The Blazhko effect does not yet have a generally accepted explanation.

As already discussed by Kovács $(1995,2001)$, one of the most plausible models to explain the Blazhko effect predicts the dependence of the Blazhko amplitude upon the strength of

Send offprint requests to: $\mathrm{M}$. Chadid, e-mail: chadid@obs-nice.fr

* Based on observations obtained with the MuSiCoS spectropolarimeter attached to the $2 \mathrm{~m}$ Bernard-Lyot Telescope of the Pic du Midi Observatory, France. a magnetic field of the order of $1 \mathrm{kG}$ in the stellar photosphere (Cousens 1983; Shibahashi \& Takata 1995; Shibahashi 1994, 2000). Unfortunately, the existence of such a magnetic field in the atmosphere of an RR Lyrae star has never been confirmed. The first search for a magnetic field in RR Lyrae was made by Babcock (1958), who reported detection of a strong longitudinal field showing variations between $B_{\mathrm{e}}=-1580$ and $+540 \mathrm{G}$ with reported probable errors of less than $220 \mathrm{G}$. However, Babcock did not find any correlation of the magnetic field behaviour with the pulsation period. Later, Preston (1967) failed to detect any magnetic field on RR Lyrae with 50 photographic observations obtained over two years (1963 and 1964). More recently, using an achromatic circular polarization analyzer, Romanov et al. $(1987,1994)$ obtained 83 Zeeman spectrograms with the $6 \mathrm{~m}$ telescope of the Russian Special Astrophysical Observatory on several nights in 1978, 1982, 1983 and 1984 with reported $1 \sigma$ uncertainties between $100-500 \mathrm{G}$. Their data appear to confirm Babcock's results; they observed a field with amplitude around $1.5 \mathrm{kG}$. They reported that the magnetic field presents a periodic variation over the pulsation cycle $(0.567 \mathrm{~d})$, while its average intensity shows a periodic long-term variation corresponding to the $40.8 \mathrm{~d}$ Blazhko period which perhaps corresponds to the stellar rotation period.

The best-studied Blazhko RR ab stars show long-term cyclical changes in their pulsation characteristics. The manifestation of these long-term changes is completely different for 
each Blazhko star. In particular, Detre \& Szeidl (1973) identified a 4-year cycle in the amplitude and phase of the Blazhko modulation of the light curve of RR Lyrae itself. This can be described as a decrease of the amplitude of the modulation and a jump in the Blazhko phase about every fourth year. In contrast, Jurcsik et al. (2002b) identified a 20-year cycle in the pulsation and Blazhko periods of another star, XZ Dra. Both Detre \& Szeidl (1973) and Jurcsik et al. (2002b) propose that this long-term cyclical behaviour would be a natural consequence of changes in the global magnetic field strength (i.e., of a magnetic cycle).

In order to evaluate the apparent detections of a magnetic field in RR Lyr by Babcock and Romanov et al., and to potentially examine the relationship between such a magnetic field and the origin of the Blazhko effect and the observed longterm cyclical modulation, we have obtained a new series of high-precision circular polarization spectroscopic observations of RR Lyrae.

\section{Observations and data processing}

\subsection{High resolution spectropolarimetric observations}

Twenty-seven Stokes $V$ spectra of RR Lyrae were obtained over four years (1999, 2000, 2001 and 2002) at various pulsation and Blazhko phases using the MuSiCoS spectropolarimeter mounted at the $2 \mathrm{~m}$ Bernard Lyot telescope at Pic du Midi Observatory. The technical characteristics of the MuSiCoS polarimeter are described in detail by Donati et al. (1997). A complete Stokes $V$ exposure consisted of a sequence of four subexposures, between which the retarder was rotated by $90^{\circ}$. All spectra were reduced using the ESpRIT reduction package (Donati et al. 1997) and cover a $210 \mathrm{~nm}$ spectral window between about 450 and $660 \mathrm{~nm}$, with an average resolving power of about 35000 . Total exposure times for a full Stokes $V$ exposure (4 subexposures) was typically 40-60 min, leading to signal-to-noise ratios $(S / N)$ in the range 600-1000.

The Least-Squares Deconvolution (LSD, Donati et al. 1997) multiline analysis procedure was employed to extract high-precision mean Stokes $I$ and $V$ profiles from each spectrum. The line mask exployed for extraction of the RR Lyrae LSD profiles was computed using a VALD (Piskunov et al. 2002) extract stellar line list with $T_{\text {eff }}=6250 \mathrm{~K}$, $\log g=3.0$ and a solar abundance table. The RR Lyrae line mask contained 668 metallic lines with predicted central depths greater than $10 \%$ of the continuum within the spectral range 448-662 nm. The typical $S / N$ of the LSD Stokes $V$ profiles is about 3000:1. No significant circular polarization is detected (according to the Shorlin et al. 2002 criterion) in any of our RR Lyrae LSD Stokes $V$ profiles.

To confirm the correct operation of the MuSiCoS instrument during RR Lyrae observations, we obtained simultaneously 9 observations of the prototypical magnetic Ap standard star $\alpha^{2} \mathrm{CVn}$. Data were reduced in the same manner as the RR Lyrae spectra, and the LSD profiles were extracted using an A0p line mask (see Wade et al. 2000a). The $S / N$ ratio in the $\alpha^{2}$ CVn Stokes $V$ LSD profiles is typically around 5000:1. The mean Landé factor corresponding to all lines in the A0p line
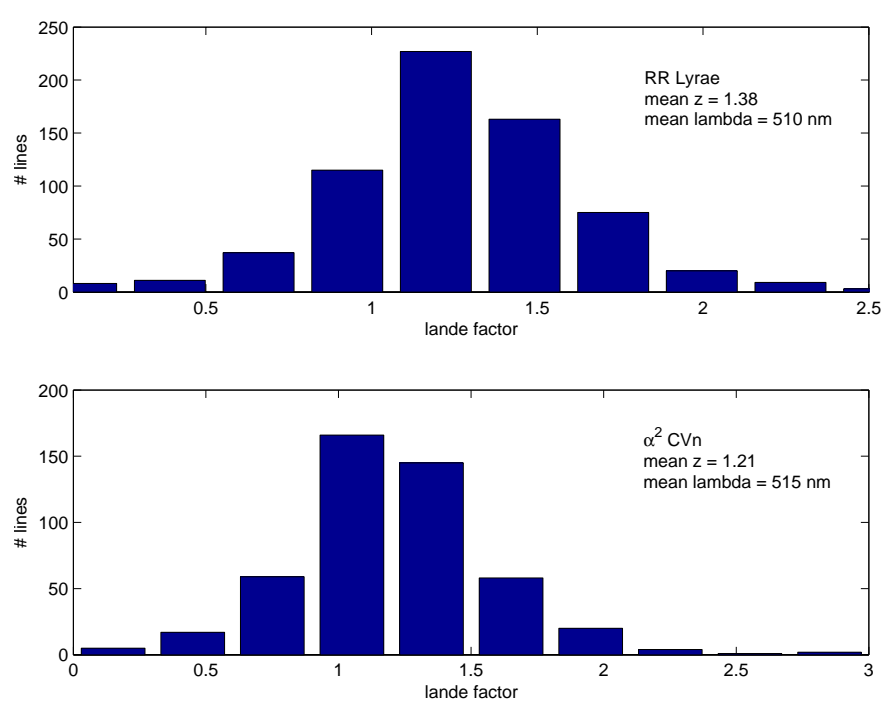

Fig. 1. Distribution of Landé factors of all lines used to construct the LSD profile of RR Lyrae (top) and $\alpha^{2} \mathrm{CVn}$ (bottom).

mask, and therefore to the $\alpha^{2} \mathrm{CVn}$ LSD profiles, is 1.21. The distribution of Landé factors is shown in Fig. 1, and compared with the analogous distribution for RR Lyrae. In constrast to RR Lyrae, significant circular polarization is detected in every single LSD Stokes $V$ profile of $\alpha^{2} \mathrm{CVn}$.

The journals of observations for both RR Lyrae and $\alpha^{2} \mathrm{CVn}$ are reported in Tables 1 and 2 .

\subsection{Spectropolarimetric measurements of Stokes $V$ and longitudinal magnetic field}

The longitudinal magnetic field values and the associated uncertainties were inferred from the extracted LSD Stokes $I$ and $V$ Zeeman profiles sets by numerical integration, using the expression:

$B_{\ell}(G)=-2.14 \times 10^{11} \frac{\int v V(v) \mathrm{d} v}{\lambda g c \int\left[I_{\mathrm{c}}-I(v)\right] \mathrm{d} v}$

(Donati et al. 1997; Wade et al. 2000b) where $c$ is the velocity of light (in the same units as $v$ ), $\lambda$ (in nm) and $g$, the mean wavelengh, and the mean value of the Lande factors of all lines used to construct the LSD profile. For RR Lyrae, these quantities are equal to $510 \mathrm{~nm}$ and 1.38 , respectively. The distribution of Landé factors of lines in the RR Lyrae line mask is compared with the analogous distribution for $\alpha^{2} \mathrm{CVn}$ in Fig. 1. The accuracy of this technique for determining high-precision longitudinal field measurements has been clearly demonstrated by Wade et al. (2000b), Donati et al. (1997), Shorlin et al. (2002) and Wade et al. (2002). The inferred values for the longitudinal magnetic fields of RR Lyrae and $\alpha^{2} \mathrm{CVn}$ are reported in the journals of observations (Tables 1 and 2).

For RR Lyrae, the Blazhko and pulsation phases have been calculated respectively from the ephemerides given by Chadid \& Gillet (1997):

$\mathrm{HJD}($ max.light ampl. $)=2449631.312+40.8 \mathrm{E}($ Blazhko $)$ and $\mathrm{HJD}(\max$. light $)=2446654.368+0.566839 \mathrm{E}($ light $)$. 


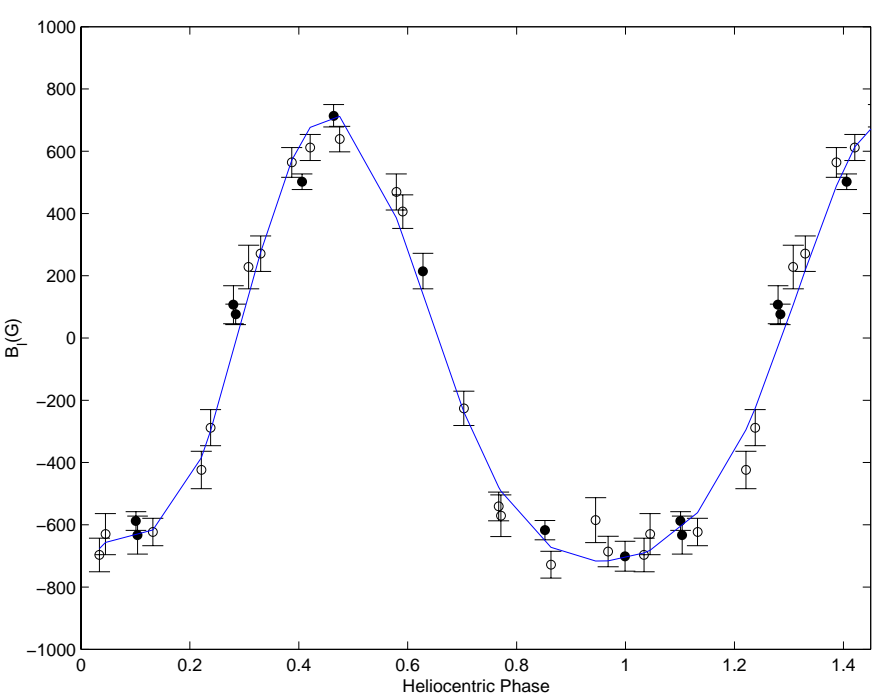

Fig. 2. Longitudinal magnetic field variation of the prototypical magnetic Ap star $\alpha^{2} \mathrm{CVn}$. The open and solid circles show respectively the observations reported by Wade et al. (2000b) and the longitudinal magnetic field measurements obtained within the context of this programme. The solid curve is a least-squares fit.

The maximum pulsation and Blazhko phases correspond respectively to $\phi=0.00$ and $\psi=0.00$. The longitudinal field measurements of RR Lyrae are by far the best ever obtained, with $1 \sigma$ uncertainties between $33-144 \mathrm{G}$, and a median value of $80 \mathrm{G}$. The strongest longitudinal field measured is $B_{\ell}=$ $+280 \pm 107 \mathrm{G}$, obtained at minimum Blazhko phase, whereas the smallest is $B_{\ell}=-5 \pm 53 \mathrm{G}$, also obtained at minimum Blazhko phase. None of our measurements of RR Lyrae represents a significant detection - the most significant measurement, as characterised using the $z=\left|B_{\ell} / \sigma_{\mathrm{B}}\right|$ parameter, has $z=2.60$.

The longitudinal field measurements of $\alpha^{2} \mathrm{CVn}$ were obtained to test the nominal functioning of the MuSiCoS instrument. All the $B_{\ell}$ measurements of $\alpha^{2} \mathrm{CVn}$ were phased according to the ephemeris of Farnsworth (1932):

$\mathrm{JD}=2419869.720+5.46939 \mathrm{E}$

and these phases are reported in Table 2 . The $B_{\ell}$ measurements of $\alpha^{2} \mathrm{CVn}$ are very precise, have uncertainties in the range 25-61 G, and confirm totally the field variability inferred by Wade et al. (2000b) (see Fig. 2). For comparison with the measurements of RR Lyrae, the most significant measurement of $\alpha^{2} \mathrm{CVn}$ has $z=20.6$ (corresponding to the longitudinal field $B_{\ell}=-617 \pm 31 \mathrm{G}$ on HD 2452488.3538 ).

\section{Discussion}

\subsection{Is RR Lyrae a magnetic star?}

Romanov et al. (1987, 1994) obtained 83 circular polarization observations of RR Lyrae on several nights in 1978, 1982, 1983 and 1984, with $1 \sigma$ uncertainties between 100-500G. According to these authors, RR Lyrae exhibits a longitudinal magnetic field which varies approximately sinusoidally,
Table 1. Journal of MuSiCoS spectropolarimetric observations of RR Lyrae at the TBL. The heliocentric Julian Date is given at midexposure. The following two columns list the pulsation and Blazhko phases. The last column lists the derived longitudinal magnetic field values and their corresponding uncertainties.

\begin{tabular}{lcccr}
\hline \hline Date & $\begin{array}{c}\text { HJD } \\
2450000+\end{array}$ & $\begin{array}{c}\text { Pulsation } \\
\text { phase }\end{array}$ & $\begin{array}{c}\text { Blazhko } \\
\text { phase }\end{array}$ & $\begin{array}{c}B_{\ell} \pm \sigma_{\mathrm{B}} \\
(\mathrm{G})\end{array}$ \\
\hline 99 Jul. 1 & 1361.4816 & 0.146 & 42.406 & $87 \pm 103$ \\
99 Jul. 3 & 1362.5145 & 0.968 & 42.431 & $114 \pm 136$ \\
99 Jul. 4 & 1363.5597 & 0.812 & 42.457 & $280 \pm 107$ \\
00 Mar. 4 & 1608.7120 & 0.302 & 48.752 & $-11 \pm 70$ \\
00 Jun. 16 & 1712.4086 & 0.241 & 51.007 & $-28 \pm 57$ \\
00 Jun. 17 & 1712.5277 & 0.451 & 51.010 & $37 \pm 44$ \\
00 Jun. 27 & 1723.4398 & 0.701 & 51.278 & $87 \pm 66$ \\
00 Jun. 28 & 1723.5545 & 0.904 & 51.280 & $59 \pm 40$ \\
00 Jun. 28 & 1724.4262 & 0.441 & 51.302 & $-47 \pm 33$ \\
00 Jun. 29 & 1724.5373 & 0.638 & 51.305 & $-8 \pm 33$ \\
00 Jul. 6 & 1732.4441 & 0.586 & 51.498 & $57 \pm 130$ \\
00 Jul. 7 & 1732.5274 & 0.733 & 51.500 & $-216 \pm 101$ \\
00 Jul. 7 & 1732.6108 & 0.880 & 51.502 & $-198 \pm 131$ \\
00 Jul. 8 & 1733.5860 & 0.600 & 51.526 & $80 \pm 144$ \\
00 Jul. 9 & 1734.5375 & 0.279 & 51.550 & $-5 \pm 53$ \\
00 Jul. 9 & 1734.6278 & 0.439 & 51.552 & $-89 \pm 46$ \\
01 Jul. 13 & 2103.5043 & 0.199 & 60.593 & $29 \pm 73$ \\
02 Jul. 06 & 2462.4108 & 0.370 & 69.390 & $-120 \pm 92$ \\
02 Jul. 06 & 2462.4677 & 0.470 & 69.391 & $21 \pm 121$ \\
02 Jul. 07 & 2463.4143 & 0.141 & 69.414 & $122 \pm 127$ \\
02 Jul. 07 & 2463.4747 & 0.247 & 69.416 & $51 \pm 103$ \\
02 Jul. 31 & 2487.6172 & 0.839 & 70.007 & $74 \pm 66$ \\
02 Aut. 01 & 2488.5165 & 0.425 & 70.030 & $73 \pm 61$ \\
02 Aut. 01 & 2488.6346 & 0.633 & 70.032 & $39 \pm 94$ \\
02 Aut. 03 & 2490.5359 & 0.987 & 70.079 & $-105 \pm 58$ \\
02 Aut. 04 & 2491.4998 & 0.688 & 70.103 & $-32 \pm 77$ \\
02 Aut. 04 & 2491.5582 & 0.791 & 70.104 & $-39 \pm 109$ \\
\hline & & & & \\
\hline & & &
\end{tabular}

Table 2. Journal of MuSiCoS spectropolarimetric observations of $\alpha^{2} \mathrm{CVn}$ at the TBL. The heliocentric Julian Date is given at midexposure. The following two columns list the pulsation and Blazhko phases. The last column lists the derived longitudinal magnetic field values and their corresponding uncertainties.

\begin{tabular}{lcr}
\hline \hline $\begin{array}{l}\text { HJD } \\
2450000+\end{array}$ & $\begin{array}{c}\text { rotational } \\
\text { phase }\end{array}$ & \multicolumn{1}{c}{\begin{tabular}{c}
\multicolumn{1}{c}{$B_{\ell} \pm \sigma_{\mathrm{B}}$} \\
$(\mathrm{G})$
\end{tabular}} \\
\hline 1732.3526 & 0.628 & $215 \pm 57$ \\
1734.3830 & 0.999 & $-701 \pm 48$ \\
2101.4028 & 0.104 & $-633 \pm 61$ \\
2102.3678 & 0.280 & $107 \pm 61$ \\
2103.3713 & 0.464 & $714 \pm 61$ \\
2462.3665 & 0.101 & $-588 \pm 30$ \\
2463.3664 & 0.284 & $76 \pm 33$ \\
2488.3538 & 0.852 & $-617 \pm 31$ \\
2491.3821 & 0.406 & $502 \pm 25$ \\
\hline
\end{tabular}

with a maximum value of $\sim 1800 \mathrm{G}$ and an amplitude of the field variation of $\sim 1000 \mathrm{G}$, when phased according to the pulsation period of 0.567 days. We point out that the phase of strongest positive longitudinal magnetic field obtained by Romanov et al. corresponds to the phase of primary shock wave passage $(\phi=0.95)$ just before the phase of maximum light. 


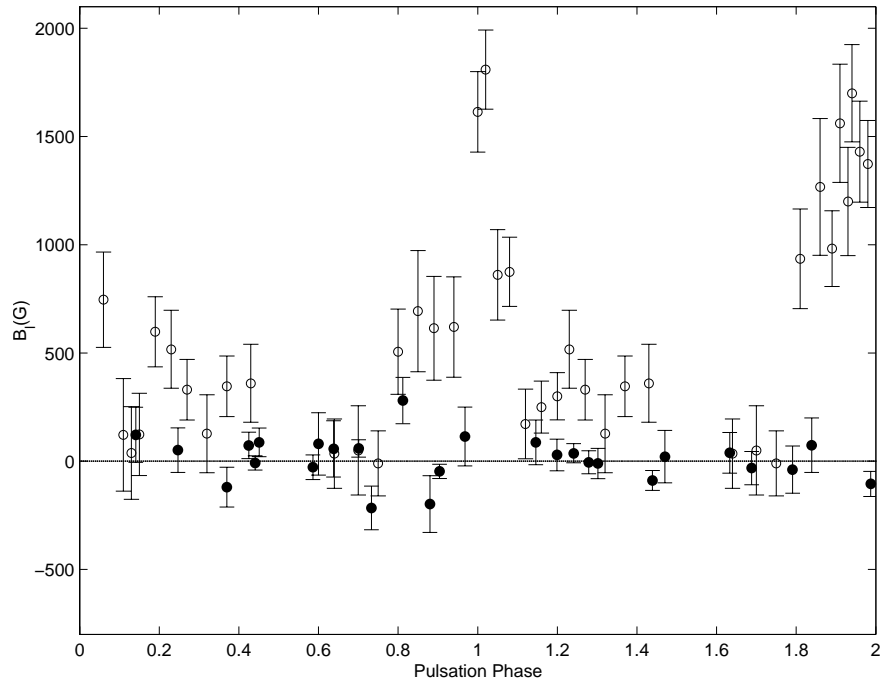

Fig. 3. Comparison of our longitudinal magnetic field measurements (solid circles) of RR Lyrae as a function of pulsation phase with the magnetic field variation reported by Romanov et al. 1987 in 25-28 September 1982 (open circles).

Figure 3 shows a comparison of our 27 RR Lyrae longitudinal magnetic field measurements with those of Romanov et al. (1987) in 25-28 September 1982, phased according to the pulsational ephemeris. It is clear that our new measurements are in strong conflict with those of Romanov et al., specially at phase $\phi=0.95$ where Romanov et al. obtain a magnetic field around 13 times larger than our typical sigma, using the mean of the Romanov et al. measurements around $\phi=0.95$.

Romanov et al. $(1987,1994)$ also claimed that the average strength and the polarity of the longitudinal field vary according to the $40.8 \mathrm{~d}$ Blazhko period: $B_{\ell}$ is strongest and positive at Blazhko phase maximum. However, as shown in Fig. 4, our measurements do not reveal any magnetic variability according to the Blazhko phase, in strong contrast with those of Romanov et al.

The absence of a significant longitudinal magnetic field in RR Lyrae is supported by another feature of the LSD technique, which is that one can detect very weak circularly polarized (Stokes $V$ ) Zeeman signatures in the LSD mean profile, even in the case where the longitudinal field is null (see Shorlin et al. 2002). For stars with non-zero $v \sin i$, this allows in principle the detection of even relatively complex magnetic field geometries. This method has been employed recently by Wade et al. (2002) (who detected no circular polarization in LSD profiles of the Cepheid star $\eta$ Aql) and by Shorlin et al. (2002) (who reported similar null results for a sample of dwarfs, subdwarfs, giants and supergiants).

In none of our RR Lyrae LSD Stokes $V$ profiles is a significant signal detected. Specifically, we find no evidence of any circular polarization (with a $Z$ upper limit of 1.5) in the mean Stokes profiles of RR Lyrae at phases corresponding to either maximum light or maximum Blazhko effect. Examples of such profiles are shown in Fig. 5.

In summary, our 27 new longitudinal magnetic field measurements of RR Lyrae are fully consistent with the hypothesis of a null longitudinal magnetic field, and provide no evidence

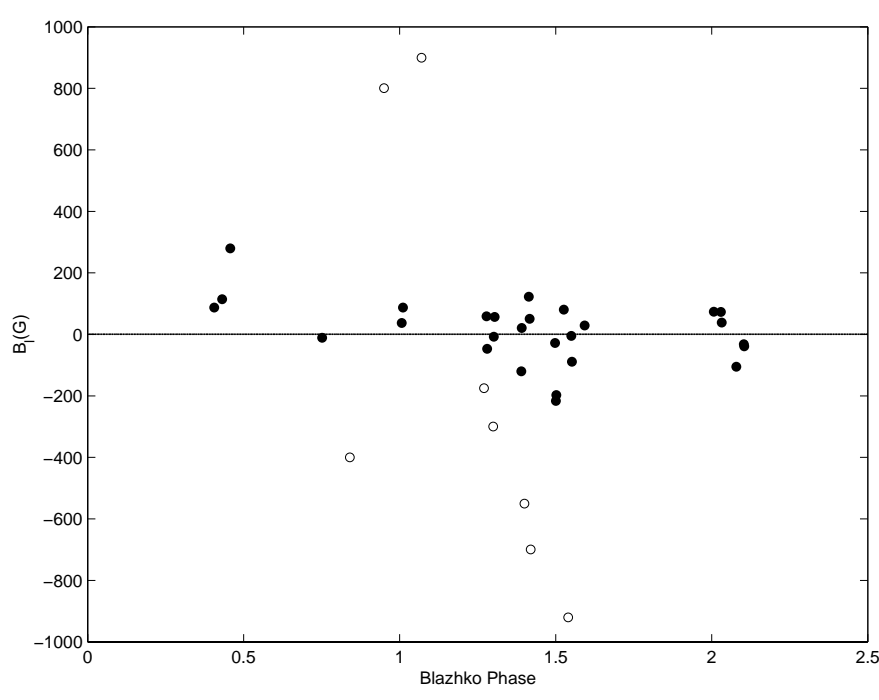

Fig. 4. Comparison of our longitudinal magnetic field measurements (solid circles) of RR Lyrae as a function of Blazhko phase with the mean magnetic field variation reported by Romanov et al. (1994) (open circles)

whatsoever of any such field during our 4 years of observations, from 1999 through 2002. The LSD mean Stokes $V$ profiles associated with each of these measurements fully support this conclusion.

How can these new results be reconciled with the apparent field detections by Babcock (1958) and Romanov et al. (1987, 1994)? The answer may lie in the fact that both Romanov et al. and Babcock employed photographic spectra in their analysis of RR Lyrae. As was pointed out by Preston (1967), uncertainties associated with longitudinal field measurement obtained using photographic spectra are frequently underestimated by factors of up to $300 \%$. This phenomenon is supported explicitly by the results of Wade et al. (2000), and implicitly by the large values of the $\chi^{2}$ parameter obtained by Wade et al. (2000) for photographic measurements obtained using the Main Stellar Spectrograph at the Russian Special Astrophysical Observatory. We point out that this same telescope and instrument were employed by Romanov et al. $(1987,1994)$ to obtain their data. Therefore it would not be surprising if their uncertainties were underestimated by a factor of at least 2 . This would render their detection marginal. Even if we allow this marginal detection, we need to consider possible sources of systematic error. As is described by Wade et al. (2002), magnetic measurements of pulsating stars can be complicated by the passage of shock waves through the stellar atmosphere. In RR Lyrae, two strong shock waves are observed (Chadid \& Gillet 1996a,b): the first around pulsation phase 0.95 (the primary shock originating from the $\kappa$-mechanism expansion) and a second weaker shock around phase 0.65 (the "rebound" shock). These shocks can lead to rapid differential changes in the line profiles (broadening and doubling phenomena), and may result in spurious polarization signatures. Therefore the correspondence between the passage of the primary shock wave and the peak of $B_{\ell}$ as obtained by Romanov et al. (1987), rather than indicating the presence of a strong magnetic field 


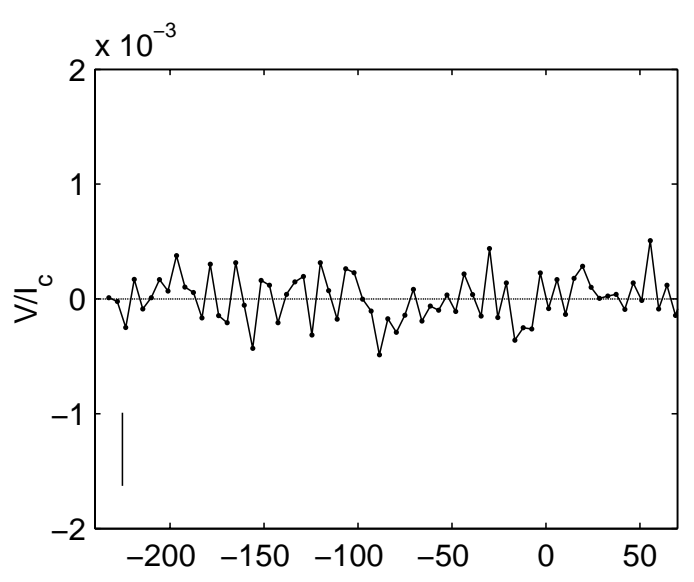

(a)

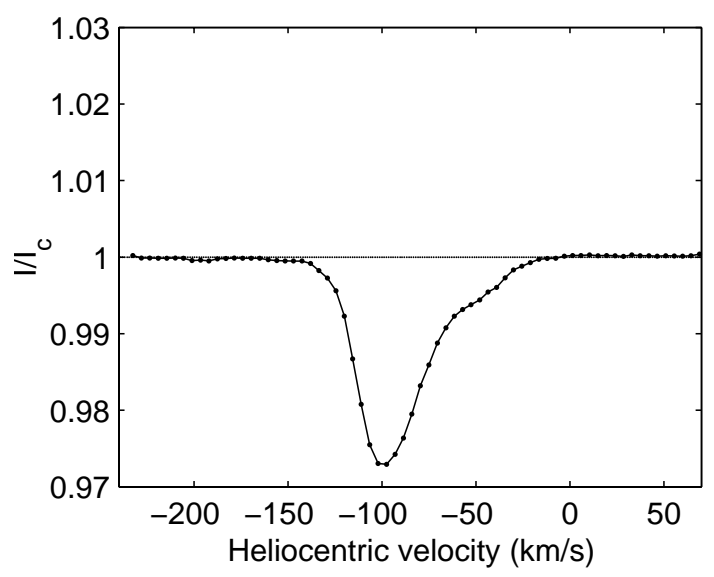

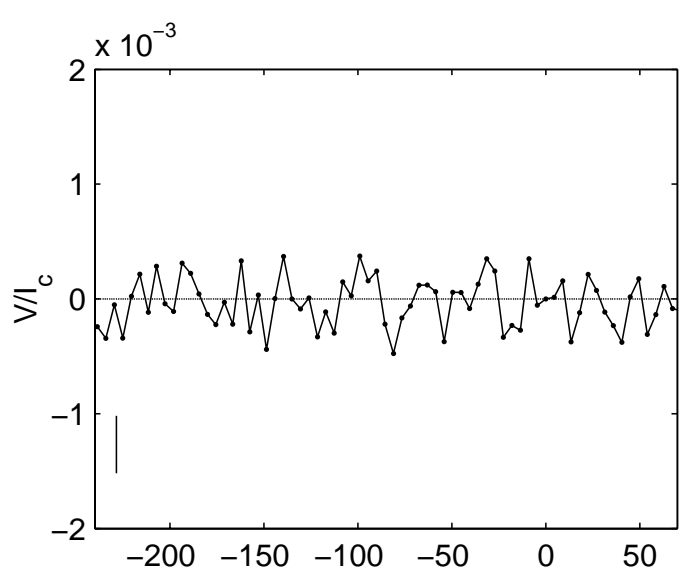

(b)

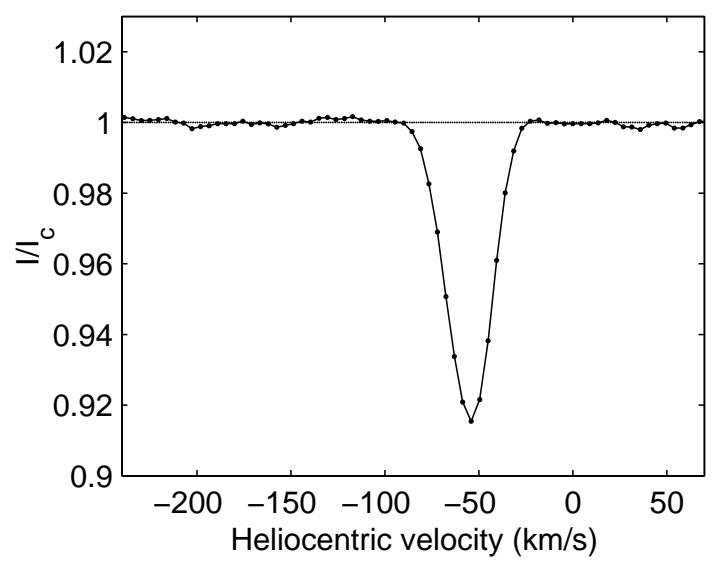

Fig. 5. a) and b) show respectively the representative LSD unpolarized Stokes $I$ (lower curves) and circularly polarized Stokes $V$ (upper curves) profiles of RR Lyrae. a) LSD $V$ and $I$ profiles obtained on 31 July 2002 corresponding to maximum pulsation phase $(\phi \sim 0.00)$ and maximum Blazhko phase $(\psi \sim 0.00)$, the heliocentric radial velocity value is $-99.24 \mathrm{~km} \mathrm{~s}^{-1}$ and the line broadening phenomenon associated with the shock wave passage is clearly visible (Chadid \& Gillet 1996b). b) LSD $V$ and $I$ profiles obtained on 9 July 2000 corresponding to minimum pulsation phase $(\phi \sim 0.50)$ and minimum Blazhko phase $(\psi \sim 0.50)$, the heliocentric radial velocity value is $-55.40 \mathrm{~km} \mathrm{~s}^{-1}$. Significant Zeeman signature is not detected in either a) or b).

at this phase, may simply indicate important spurious contributions to the Stokes $V$ profiles due to shock-related variations.

Although we are unable to completely dismiss the apparent detections of magnetic fields in RR Lyrae by Babcock (1958) and Romanov et al. (1987, 1994), we propose that: (1) the lack of detection of a field by Preston (1967), (2) the potential of the Babcock and Romanov data for underestimated uncertainties and important spurious polarization signatures, (3) the lack of detection of any significant magnetic field from four years of high-precision circular polarization measurements with the MuSiCoS spectropolarimeter, and (4) the simultaneous MuSiCoS detection of a magnetic field in the magnetic standard star $\alpha^{2} \mathrm{CVn}$, suggest very strongly that RR Lyrae is not a magnetic star.

\subsection{Is there any long term magnetic activity of RR Lyrae?}

The Blazhko star RR Lyrae also displays a long period cycle, besides the radial pulsation and the Blazhko cycle.
Detre \& Szeidl (1973) identified a four-year cycle in the amplitude and phase of the Blazhko modulation of the light curve of RR Lyrae. The origin of the long-term cycle has not been established. In contrast, Jurcsik et al. (2002b) identified a 20-year cycle in the pulsation and Blazhko periods of Blazhko star XZ Dra. Like Stothers's scenario (1980), both Szeidl (1973) and Jurcsik et al. (2002b) explain that these long-term modulations of RR Lyrae and XZ Dra seem to be a natural consequence of changes in the global magnetic field strength and structure during the magnetic cycle wich is 4-year for RR Lyrae and 20-year for XZ Dra. Stothers (1980) proposes also that the apparent absence of the Blazhko effect would be due to the analog of the long minimum of the much less regular 80-year cycle of the sun. He explains so that the RR Lyrae stars without Blazhko effect could be understood as a temporary cessation of a strong magnetic activity. In order to check the above mentionned hypothesis, we plotted in Fig. 6. our longitudinal magnetic field measurements of RR Lyrae along our four year observations (1999-2002). Once again, we point out that no clear correlation of the magnetic field behaviour with the period of about 4 years. Therefore, we do not support the 


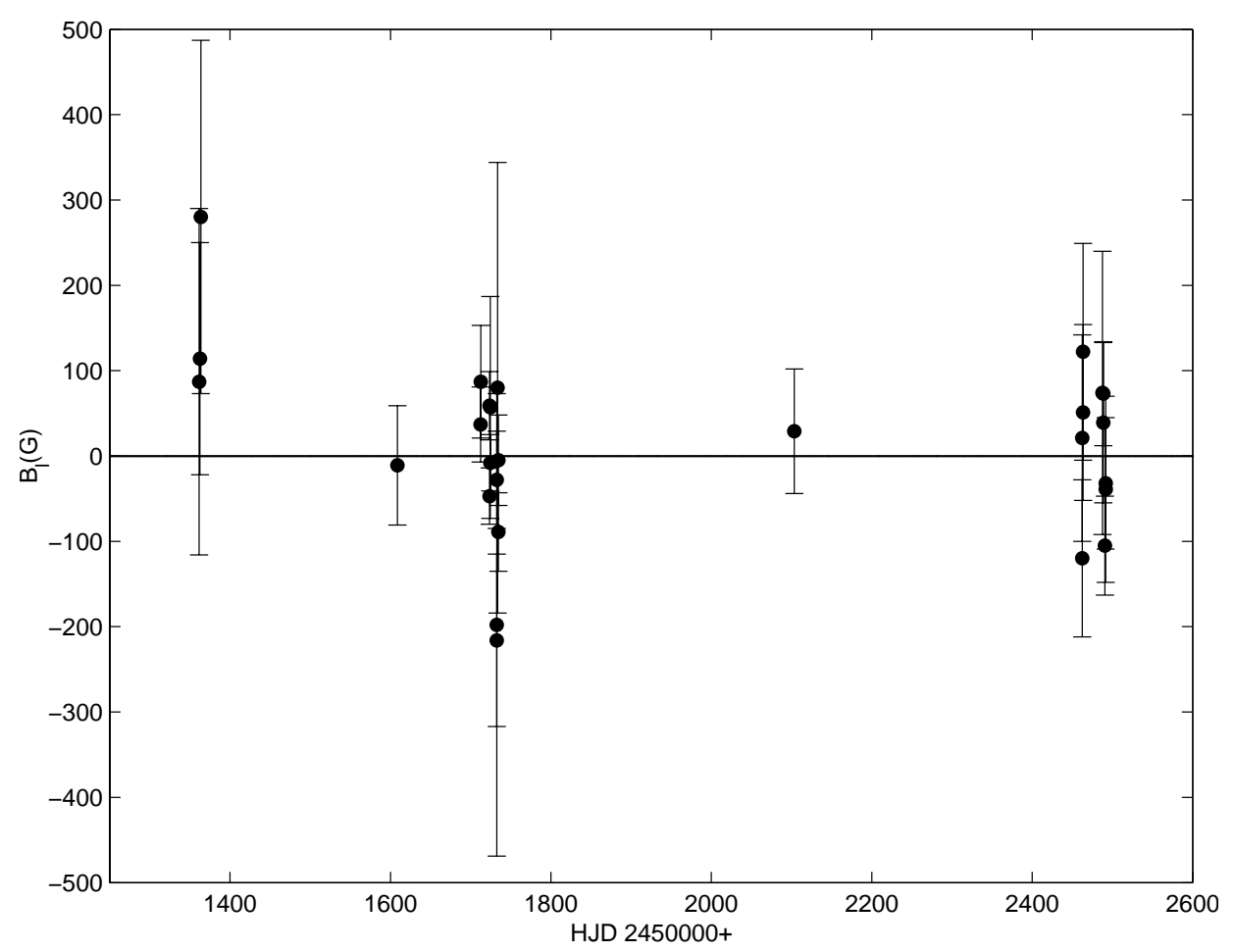

Fig. 6. Longitudinal magnetic field measurements of RR Lyrae from 1999 through 2002. No clear magnetic field variability is visible with the period of about 4 years.

magnetic cycle hypothesis to explain the origin of the existence of the long-term modulation in RR Lyrae. However, a new detailed theoretical work is needed to the understanding of the origin of the long-term modulation.

\subsection{How can we explain the Blazhko effect?}

Until now, the most plausible hypotheses to explain the Blazhko phenomenon focus on two types of models, both identifying the Blazhko period as the rotational period, and both involving nonradial pulsational modes. Such nonradial modes were detected for the first time by Chadid et al. (1999), who pointed out the existence of a triplet (and possibly quintuplet) structure of RR Lyrae's frequency spectrum.

The first model type is the resonance models, in which there exists a nonlinear resonance between the dominant radial fundamental mode and a nonradial mode. In these models the dipole mode $(l=1)$ has the highest probability to be nonlinearly excited (Cox 1993; Van Hoolst et al. 1998; Nowakowski \& Dziembowski 2001). The dipole pulsation components lead to a triplet structure in the frequency spectrum of Blazhko variables. In the resonance model proposed by Nowakowski \& Dziembowski (2001), significant amplitude and phase modulation of the light curve is predicted from the excitation of a rotationally split $m= \pm 1$ pair and the modulation period is determined by the rotation rate and the Brunt-Vaisala frequency in the deepest part of the radiative envelope. It should be noted that this model is not able to explain possible changes in the period of the Blazhko effect that have been reported for XZ Dra (Jurcsik et al. 2002b), RW Dra (Firmanyuk 1978), RV UMa (Kanyó 1976), and XZ Cyg (LaCluyze et al. 2002).
The second type of model is the magnetic models, analogous to the oblique pulsator model for rapidly oscillating roAp stars (Kurtz 1982). These models suppose that Blazhko stars have a strong magnetic field of order $1 \mathrm{kG}$, which is inclined relative to the stellar rotational axis (Cousens 1983; Shibahashi \& Takata 1995; Shibahashi 1994, 2000). According to these models, the interpretation of the Blazhko effect is as follows: the radial eigenfunction excited by the $\kappa$ mechanism is deformed, primarily due to the Lorentz force, providing an additional quadrupole component $(l=2)$ whose symmetry axis coincides with the magnetic axis. As the star rotates, the aspect angle of this non-radial pulsation mode varies and these components manifest themselves as the long term modulation of RR Lyrae stars. Shibahashi \& Takata (1995) predict a quintuplet frequency structure (may appear to be a triplet for certain geometrical configurations) and they suppose that the Blazhko period is the rotational period. The current generation of magnetic models, such as the oblique pulsator model (Cousens 1983; Shibahashi \& Takata 1995; Shibahashi 1994, 2000) require a strong magnetic field of the order of $1 \mathrm{kG}$ to explain the Blazhko modulation.

Moreover, it seems difficult to select which model resonance and/or magnetism is able to explain the Blazhko effect, because it has still been hard to identify exactly which nonradial pulsation modes are excited in RR Lyrae based only on photometric observations (Smith et al. 2003) or on spectroscopic observations poorly spread over the Blazhko cycle (Chadid et al. 1999).

Our recent observations showing the absence of magnetic field in RR Lyrae reject the magnetic models as a plausible hypothesis to explain the Blazhko effect. 


\section{Conclusions}

Using twenty-seven new high-precision circular polarization measurements, obtained over a period of almost 4 years and within a median $1 \sigma$ longitudinal magnetic field uncertainty of $80 \mathrm{G}$, we fail to detect any evidence of a magnetic field in the photosphere of RR Lyrae. These results strongly contradict apparent field detections by Babcock (1958) and Romanov et al. (1987, 1994), but agree with the result of Preston (1967). We suggest that the reported detections are in fact spurious, resulting from the combination of underestimated error bars and spurious signatures caused by rapid line profile variations during phases of shock wave passage. We conclude that RR Lyrae is in fact a bona fide non-magnetic star. Our observations are further capable of ruling out the "magnetic-cycle" hypothesis which propose that RR Lyrae undergoes a solar cycle-type field oscillation with a period of about 4 years.

The undermining of magnetic models as a possible source of the Blazhko effect implies that renewed investigation of other models must be undertaken.

Acknowledgements. This work has taken advantage of the Vienne Atomic Line Database (VALD) and the SIMBAD database, operated at CDS Strasbourg, France. We would like to thank the referee Dr. H. Shibahashi for very constructive comments and suggestions, and express our gratitude to Prof. J. Vernin for fruitful discussions. GAW and JDL acknowledge support from the Natural Science and Engineering Research Council of Canada (NSERC) in the form of Research Grants.

\section{References}

Babcock, H. M. 1958, ApJS, 3, 141

Blazhko, S. 1907, Astr. Nachr., 175, 325

Chadid, M., \& Gillet, D. 1996a, A\&A, 308, 481

Chadid, M., \& Gillet, D. 1996b, A\&A, 315, 475

Chadid, M., \& Gillet, D. 1997, A\&A, 319, 154

Chadid, M., Kolenberg, K., Aerts, C., \& Gillet, D. 1999, A\&A, 352, 201

Chadid, M. 2003, A\&A, in preparation

Cousens, A. 1983, MNRAS, 203, 1171

Cox, A. N. 1993, New Perspectiveson Stellar Pulsation and Pulsating Stars, ed. J. M. Nemec, \& J. M. Matthews, Proc. IAU Coll., 139, 409

Detre, L., \& Szeidl, B. 1973, in Variable Stars in Globular Clusters and related Systems, ed. J. D. Fernie, IAU Coll., 21, 31

Donati, J. F., Semel, M., Carter, B.D, \& Cameron, A. C. 1997, MNRAS, 291, 658

Donati, J. F., Catala, C., Wade, G. A., et al. 1999, A\&AS, 134, 149
Donati, J. F., Wade, G. A., Babel, J., et al. 2001, MNRAS, 326, 1265

Firmanyuk, B. N. 1978, Astron. Circ., 1019, 3

Jurcsik, J., Benko, J. M., \& Szeidl 2002a, A\&A, 390, 133

Jurcsik, J., Benko, J. M., \& Szeidl 2002b, A\&A, 396, 539

Kanyó, S. 1976, Commun Konkoly Obs. Budapest, 7(69), 1

Kovacs, G. 1995, A\&A, 295, 693

Kovacs, G. 2001, Stellar pulsation-nonlinear studies, ed. M. Takeuti, \& D. D. Sasselov, Astrophys. Space Sci. Lib., 257, 61 (Dordrecht: Kluwer Academic Publishers), ISBN 0-7923-6818-5

Kurtz, D. W. 1982, MNRAS, 200, 807

LaCluyz, A., Smith, H. A., Gill, E. M., et al. 2002, Radial and Nonradial Pulsations as Probes of Stellar Physics, ed. C. Aerts, T. R. Beddings, \& J. Christensen-Dalsgaard, ASP Conf. Proc., 259,416

Landstreet, J. D. 1988, ApJ, 326, 967

Nowakowski, R. M., \& Dziembowski, W. A. 2001, Acta Astron., 51, 5

Piskunov, N. E., Kupka, F., Ryabchikova, T. A., Weiss, W. W., \& Jeffery, C. S. 1995, A\&AS, 112, 525

Preston, G. W. 1967, The Magnetic and Related Stars, ed. R. C. Cameron (Baltimore: Mono Book Corporation), 26

Romanov, Yu. S., Udovichenko, S. N., \& Frolov, M. S. 1987, Sov. Astron. Lett., 13, 29

Romanov, Yu. S., Udovichenko, S. N., \& Frolov, M. S. 1994, Bul. Spec. Astrophys. Obs., 38, 169

Shibahashi, H. 1994, What's new in the theory of stellar nonradial oscillations, ASP Conf. Ser., 76, 618

Shibahashi, H., \& Takata, M. 1995, ASP Conf. Ser., 83, 42

Shibahashi, H. 2000, The oblique pulsator model for the Blazhko effect in RR Lyrae stars, Theory of amplitude modulation I, ASP Conf. Ser., 203, 299

Shorlin, S. L. S., Wade, G. A., Donati, J. F., et al. 2002, A\&A, 392, 637

Smith, H. A., Church, J. A., Fournier, J., et al. 2003, PASP, 115, 43

Stothers, R. 1980, PASP, 92, 475

Szeidl, B. 1976, in Multiple Periodic Variable Stars, ed. W. S. Fitch (Budapest: Akademia), IAU Colloq., 29, 133

Van Hoolst, T., Dziembowski, W. A., \& Kawaler, S. D. 1998, MNRAS, 297, 536

Wade, G. A., Elkin, V. G., Landstreet, J. D., et al. 1996, A\&A, 313, 209

Wade, G. A., Elkin, V. G., Landstreet, J. D., \& Romanyuk, I. I. 1997, MNRAS, 292, 748

Wade, G. A., Donati, J. F., Landstreet, J. D., \& Shorlin, S. L. S. 2000a, MNRAS, 313, 823

Wade, G. A., Donati, J. F., Landstreet, J. D., \& Shorlin, S. L. S. 2000b, MNRAS, 313, 851

Wade, G. A., Bagnulo, S., Kochukhov, O., et al. 2001, A\&A, 374, 265

Wade, G. A., Chadid, M., Shorlin, S. L. S., Bagnulo, S., \& Weiss, W. 2002, A\&A, 396, L17

Wilson, P. R. 1994, Solar and Stellar Activity Cycles, Cambridge astrophysics series No. 24 (Cambridge: Cambridge University Press) 